\title{
EXAMPLES OF PERIODIC MAPS ON EUCLIDEAN SPACES WITHOUT FIXED POINTS
}

\author{
BY J. M. KISTER ${ }^{1}$
}

Communicated by Deane Montgomery, March 29, 1961

Let $T$ be a map of period $r$ on a Euclidean space $E^{n}$. Smith seems to have been the first to consider fixed points of $T$. He showed that $T$ has a fixed point if $r$ is a prime in [4], extended this result to $r$ a power of a prime, and raised the question concerning the existence of a fixed point for $r$ not a prime power in [5]; also cf. Problem 33 in [3]. Conner and Floyd gave an example of a contractible manifold $M_{r}$ for every $r$ not a prime power, and a map $T$ of period $r$ on $M_{r}$ without fixed points [2]. They conjectured that $M_{r}$ was a Euclidean space. This note shows that a slight modification of their example is Euclidean, hence:

THEOREM. If $r$ is an integer which is not a power of a prime, then there exists a triangulation $\tau$ of $E^{9 r}, a$ map $T$ of period $r$ on $E^{9 r}$ without fixed points, and $T$ is simplicial relative to $\tau$.

I wish to express my indebtedness to Professor Floyd for his help and encouragement.

Preliminaries. Let $K$ be a subcomplex of a Euclidean space $E$ under a triangulation $\sigma$. Let $\sigma_{K}^{(1)}$ be the subdivision of $\sigma$ obtained by adding barycenters of all simplexes not contained in $K$, cf. [6, p. 251]. $\sigma_{K}^{(i+1)} \equiv\left(\sigma_{i K}^{(i)}\right)_{K}^{(i)}$. If $K$ is the empty complex, $\sigma_{K}^{(i)} \equiv \sigma^{(i)}$, the usual $i$ th barycentric subdivision. Denote the closed star of $K$ in $\sigma$ by $V(K, \sigma)$ and let $V^{2}(K, \sigma)=V(V(K, \sigma), \sigma) . N_{W}(K, \sigma) \equiv V\left(K, \sigma_{K}^{(2)}\right)$ is a "regular" neighborhood of $K$; cf. [6, p. 293]. If $K$ is a contractible finite subcomplex having dimension $m$ and $E=E^{n}$, where $n \geqq 2 m+5$, then it follows from Corollary 3 in $\left[6\right.$, p. 298] that $N_{W}(K, \sigma)$ is an $n$-cell. Much use is made of this fact; however it will be convenient later to use the following neighborhood: $N_{1}(K, \sigma) \equiv V\left(K^{(2)}, \sigma^{(2)}\right)$, i.e. the star of $K$ (subdivided twice barycentrically) in $\sigma^{(2)}$. Since it will be necessary to use Whitehead's result, but only in a topological way (i.e. noncombinatorial), it suffices to show that $N_{W}(K, \sigma)$ and $N_{1}(K, \sigma)$ are homeomorphic. This can be done by looking at an $n$-simplex $\rho$ in the triangulation $\sigma_{K}^{(1)}$ which intersects $K$, and constructing a canonical homeomorphism of $N_{W}(K, \sigma) \cap \rho$ and $N_{1}(K, \sigma) \cap \rho$ in such a way that two such homeomorphisms match on $p$-faces, $p<n$. Let $\rho=\rho_{0} \circ \rho_{1}$,

\footnotetext{
1 The author holds an ONR Research Associateship at the University of Virginia.
} 
the join, where $\rho_{0}$ and $\rho_{1}$ are simplexes in $\sigma_{R}^{(1)}$ with $\rho_{0} \cap K=\varnothing$ and $\rho_{1} \subseteq K$. It is easy to show that every segment $A_{x_{0}, x_{1}}=\left\{x_{t}=(1-t) x_{0}\right.$ $+t x_{1} \mid t$ in $\left.[0,1]\right\}$ where $x_{0}$ is in $\rho_{0}, x_{1}$ in $\rho_{1}$, intersects $N_{W}(K, \sigma)$ and $N_{1}(K, \sigma)$ in a desirable way; namely there exist $t_{W}$ and $t_{1}$ in $(0,1)$ such that $A_{x_{0}, x_{1}} \cap N_{W}(K, \sigma)=\left\{x_{t} \mid t_{W} \leqq t \leqq 1\right\} \equiv A_{W}$ and $A_{x_{0}, x_{1}}$ $\cap N_{1}(K, \sigma)=\left\{x_{t} \mid t_{1} \leqq t \leqq 1\right\} \equiv A_{1}$. Now map $A_{W}$ onto $A_{1}$ linearly and do this for each pair $x_{0}, x_{1}$ in $\rho_{0}$ and $\rho_{1}$. The regular neighborhoods used here will now be $N_{1}$, and we define $N_{i+1}(K, \sigma) \equiv N_{i}\left(N_{1}(K, \sigma), \sigma^{(2)}\right)$, a subcomplex in $E$ under $\sigma^{(2 i+2)}$. Clearly if $K_{1}$ is a subcomplex under $\sigma_{1}, K_{1} \subseteq K$, and $\sigma_{1}$ refines $\sigma$, then $N_{i}\left(K_{1}, \sigma_{1}\right) \subseteq N_{i}(K, \sigma)$, for all $i$.

For a given $r$ in the theorem let $K$ be the Conner-Floyd example of a 4-dimensional, star-finite, contractible complex with $Z_{r}$ acting without fixed points [2, p. 360]. $K$ is the union of simplicial mapping cylinders $C_{i}$ whose "beginning" $B_{i}$ and "end" $E_{i}$ are 3 -spheres. $C_{i}$ is the simplicial analogy of the ordinary mapping cylinder defined, in this case, by a simplicial map $f^{*}$ of $B_{i}$ into $E_{i}$ which is inessential. Also $E_{i}=B_{i+1}$. For a more exact description the reader is referred to [2]. It will also be assumed that $K$ is imbedded as a subcomplex of $E=E^{9 r}$ with triangulation $\sigma, S$ is a simplicial map of $E$ onto itself of period $r$ (in fact, $S\left(x_{1}, \cdots, x_{r}\right)=\left(x_{2}, \cdots, x_{r}, x_{1}\right)$ for $\left.x_{i} \in E^{9}\right), K$ is an invariant set under $S$, and $S \mid K$ is the generator of $Z_{r}$. This, too, was done in [2]. $\sigma$ can be taken fine enough so that $V\left(C_{i}, \sigma\right)$ and $V\left(C_{j}, \sigma\right)$ are disjoint if $|i-j|>1$. Since $S$ is simplicial and $K$, invariant, $N_{i}(K, \sigma)$ is invariant for each $i$, hence $E^{\prime} \equiv \bigcup_{i=1}^{\infty} N_{i}(K, \sigma)$ is invariant. Since $S$ has no fixed points in $K$ and $E^{\prime} \subseteq$ Int $V(K, \sigma), S$ has no fixed points in $E^{\prime}$. It will be shown that $E^{\prime}$ is Euclidean. To do this it will suffice to express $E^{\prime}$ as the union of cubes $\left\{I_{j}\right\}_{j=1}^{\infty}$ with $I_{j} \subseteq$ Int $I_{j+1}$ and then one could use a recently announced result of M. Brown. ${ }^{2}$ However, in this special case, where each $I_{j}$ is in $E^{n}$, $\bigcup_{j=1}^{\infty} I_{j}$ is seen to be Euclidean by an easy application of another result of Brown which is in the literature [1]. For by the characterization of a tame $S^{n-1}$ in $E^{n}$ given there, and by taking $I_{j}$ to be a slightly smaller concentric cube, there is no loss of generality in assuming that $\mathrm{Bd} I_{j}$ is a tame $S^{n-1}$ in $E^{n}$ for each $j$. Hence the complement $J$ of $\bigcup_{j=1}^{\infty} I_{j}$ in the one point compactification of $E^{n}$ is the intersection of decreasing cubes, i.e. cellular [1], hence $E^{n} \approx S^{n}-J$ $=\left(E^{n} \cup \infty\right)-J=\bigcup_{j=1}^{\infty} I_{j}$.

Lemma. Given any positive integer $i$ there exists a subdivision $\sigma_{i}$ of $\sigma$ and a finite contractible complex $K_{i}$ in $\sigma_{i}$ such that

2 The monotone union of open $n$-cells is an open n-cell, Notices Amer. Math. Soc. vol. 7 (1960) p. 478. 
(1) $L_{i} \equiv \cup_{j \leq i} C_{j} \subseteq K_{i}$.

(2) $\sigma_{i}$ agrees with $\sigma$ on $V\left(L_{i}, \sigma\right)$, hence $N_{j}\left(L_{i}, \sigma\right) \subseteq N_{j}\left(K_{i}, \sigma_{i}\right)$, for all $j$.

(3) $N_{j}\left(K_{i}, \sigma_{i}\right) \subseteq N_{j}\left(L_{i+2}, \sigma\right)$, for all $j$.

Proof. Note that if $D$ is a 4-cell and $h$ is a homeomorphism from the $\mathrm{Bd} D$ onto the end $E_{i+1}$ of $C_{i+1}$, then since $E_{i+1}$ is a strong deformation retract of $C_{i+1}$, hence of $L_{i+1}$, it follows that the identification space $L_{i+1} \cup D / h$ is contractible. The proof of the lemma depends on getting a simplicial representation $K_{i}$ of this identification space close to $L_{i+2}$.

First we produce a map $f$ of $D$ into $C_{i+2}$. For a simplicial model of $D$ take the cone over the simplicial 3-sphere $B$, where $B$ is a copy of $B_{i+2}$, the beginning of $C_{i+2} . D=\{(b, t) \mid b \in B, t \in I\} / B \times 1$. Let $D^{\prime} \equiv\{(b, t) \in D \mid t \in[0,1 / 2]\}, D^{\prime \prime} \equiv\{(b, t) \in D \mid t \in[1 / 2,1]\} . D^{\prime} \cap D^{\prime \prime}$ $\equiv B^{\prime}$. Now map $D^{\prime}$ into $C_{i+2}$ by $f^{\prime}$ such that $f^{\prime} \mid B: B \rightarrow B_{i+2}$ is a simplicial isomorphism, $f^{\prime}\left(B^{\prime}\right) \subseteq E_{i+2}$ and $f^{\prime} \mid B^{\prime}$ is inessential (it can be taken to be, essentially, $\left.f^{*}\right)$. Hence there is a map $f^{\prime \prime}: D^{\prime \prime} \rightarrow E_{i+2}$ such that $f^{\prime \prime}\left|B^{\prime}=f^{\prime}\right| B^{\prime}$. Then $f \mid D^{\prime}=f^{\prime}$ and $f \mid D^{\prime \prime}=f^{\prime \prime}$ define $f$.

Let $\epsilon$ be so small that the $\epsilon$-neighborhood (under the usual metric for $E$ ) of $C_{i+2}$ is contained in $N_{1}\left(C_{i+2}, \sigma\right)$. Since $\operatorname{dim} E=9 r>8$ we can get $g$, an $\epsilon$-approximation of $f$, which imbeds $D$ in $E$ (hence in $\left.N_{1}\left(C_{i+2}, \sigma\right)\right)$ and such that

(a) $g|B=f| B$ and $g(D) \cap L_{i+1}=B_{i+2}=g(B)$,

(b) $g$ maps linearly (using the vector space structure of $E$ ) each simplex in the $k$ th barycentric subdivision of $D$, for some $k$.

The usual technique is used, that of subdividing $D$ so that images of simplexes under $f$ are small relative to $\epsilon$, then choosing a point near each image of a vertex (keeping fixed images of vertices in $B$ ) so that the set of all such points is in general position, and then extending the obvious vertex map linearly.

Now we get a subdivision of $V\left(C_{i+2}, \sigma\right)$ so that $g(D)$ may be regarded as a subcomplex. One way of getting this would be to regard each 4-simplex in $g(D)$ as a subsimplex of a rectilinear $n$-simplex in $E$. Consider the $(n-1)$-planes determined by the $(n-1)$-faces of such $n$-simplexes, one chosen for each 4-simplex in $g(D)$. The triangulation $\sigma$, together with this finite collection of $(n-1)$-planes, partitions $V\left(C_{i+2}, \sigma\right)$ into convex polyhedral sets which can then be triangulated. Furthermore this triangulation $\sigma_{i}$ can be taken so fine that $N_{1}\left(g(D), \sigma_{i}\right)$ $\subseteq N_{1}\left(C_{i+2}, \sigma\right)$. Now extend the triangulation $\sigma_{1}$ to all of $E$ keeping $\sigma$ on $F=\mathrm{Cl}\left(E-V^{2}\left(C_{i+2}, \sigma\right)\right)$. This can be done by triangulating the "ring" $R=\mathrm{Cl}\left(V^{2}\left(C_{i+2}, \sigma\right)-V\left(C_{i+2}, \sigma\right)\right)$ without introducing any new vertices in $V^{2}\left(C_{i+2}, \sigma\right)-V\left(C_{i+2}, \sigma\right)$. Each simplex under $\sigma$ in $R$, say $\rho$, may be regarded as a join of two simplexes $\rho_{1}$ and $\rho_{2}$ in $F$ and 
$V\left(C_{i+2}, \sigma\right)$ respectively, where $\rho_{2}$ has been subdivided under $\sigma_{i}$. Triangulate $\rho$ by taking the joins of $\rho_{1}$ with the small simplexes in $\rho_{2}$ under $\sigma_{i}$. Do this for each $\rho$ in $R$ getting a triangulation $\sigma_{i}$ of $E$ which is a subdivision of $\sigma$, which agrees with $\sigma$ on $F$. Condition (2) of the lemma follows from $V\left(L_{i}, \sigma\right) \subseteq F$.

Define $K_{i}=L_{i+1} \cup g(D)$, a contractible subcomplex under the triangulation $\sigma_{i}$. Condition (1) is clearly satisfied. Since $\sigma_{i}$ refines $\sigma, N_{j}\left(L_{i+1}, \sigma_{i}\right) \subseteq N_{j}\left(L_{i+1}, \sigma\right)$ and since $N_{1}\left(g(D), \sigma_{i}\right) \subseteq N_{1}\left(C_{i+2}, \sigma\right)$, $\left.N_{j}\left(g(D), \sigma_{i}\right) \subseteq N_{j}\left(C_{i+2}\right), \sigma\right)$ and it follows that $N_{j}\left(K_{i}, \sigma_{i}\right) \subseteq N_{j}\left(L_{i \nmid 1}, \sigma\right)$ $\cup N_{j}\left(g(D), \sigma_{i}\right) \subseteq N_{j}\left(L_{i+1}, \sigma\right) \cup N_{j}\left(C_{i+2}, \sigma\right)=N_{j}\left(L_{i+2}, \sigma\right)$, hence condition (3) is satisfied and the lemma proved.

Proof of the theorem. Using the notation of the lemma, $N_{1}\left(K_{i}, \sigma_{i}\right)$ $\approx N_{W}\left(K_{i}, \sigma_{i}\right)$ is an $n$-cell by [6], hence $N_{i}\left(K_{i}, \sigma_{i}\right)$, which can be expressed as $N_{1}\left(N_{i-1}\left(K_{i}, \sigma_{i}\right), \sigma_{i}^{(2 i-2)}\right) \approx N_{W}\left(N_{i-1}\left(K_{i}, \sigma_{i}\right), \sigma_{i}^{(2 i-2))}\right)$, is an $n$-cell, which we designate by $I_{i}$. Using the lemma we get:

$$
\begin{aligned}
N_{1}\left(L_{1}, \sigma\right) \subseteq I_{1} \subseteq N_{1}\left(L_{3}, \sigma\right) \subseteq N_{3}\left(L_{3}, \sigma\right) \subseteq I_{3} \subseteq N_{3}\left(L_{5}, \sigma\right) \\
\subseteq N_{5}\left(L_{5}, \sigma\right) \subseteq I_{5} \subseteq \ldots
\end{aligned}
$$

and $I_{2 i-1}$ is contained in the interior of $I_{2 i+1}$. Then $E^{\prime}=\bigcup_{i=1}^{\infty} N_{i}(K, \sigma)$ $=\bigcup_{i=1}^{\infty} N_{i}\left(L_{i}, \sigma\right)=\bigcup_{j=1}^{\infty} I_{2 j-1}$ is Euclidean. The map $T$ is, of course, $S \mid E^{\prime}$, and the invariant triangulation is obtained in the following way. $N_{i}(K, \sigma)$ is a complex in $\sigma^{(2 i)}$, and it is subdivided twice without subdividing $N_{i-1}(K, \sigma)$, i.e. $N_{i}(K, \sigma)$ becomes a complex in $\left(\sigma^{(2 i)}\right)_{N_{i-1}(K, \sigma)}^{2(2)}$.

Added in proof. D. R. McMillan has communicated to me an alternate (and simpler) way of producing examples in $E^{18 r}$ making use of recent results of his in Cartesian products of contractible open manifolds, Bull. Amer. Math. Soc., this issue, pp. 510-514

\section{REFERENCES}

1. M. Brown, $A$ proof of the generalized Schoenflies theorem, Bull. Amer. Math. Soc. vol. $66(1960)$ pp. 74-76.

2. P. E. Conner and E. E. Floyd, On the construction of periodic maps without fixed points, Proc. Amer. Math. Soc. vol. 10 (1959) pp. 354-360. 260.

3. S. Eilenberg, On the problems of topology, Ann. of Math. vol. 50 (1949) pp. 247-

4. P. A. Smith, $A$ theorem on fixed points for periodic transformations, Ann. of Math. vol. 35 (1934) pp. 572-578.

5. - Fixed point theorems for periodic transformations, Amer. J. Math. vol. 63 (1941) pp. 1-8.

6. J. H. C. Whitehead, Simplicial spaces, nuclei and m-groups, Proc. London Math. Soc. vol. 45 (1938) pp. 243-327.

UNIVERSITY OF MICHIGAN AND

UNIVERSITY OF VIRGINIA 